\title{
CUANTIFICACIÓN DE POLIFENOLES EN HOJAS DE UN CLON DE Uncaria tomentosa (WILLD. EX SCHULT) D.C., PROVENIENTE DE TRES LOCALIDADES DE LA REGIÓN UCAYALI
}

\author{
Ruth S. Romero ${ }^{a^{*}}$, Gilberto Domínguez ${ }^{a}$, Deysi R. Guzmán ${ }^{a}$ \\ RESUMEN
}

Uña de gato (Uncaria tomentosa Willd. ex D.C.), de la familia Rubiaceae, es un arbusto que en el bosque toma forma de liana y del cual se utiliza, tradicional y comercialmente la corteza, debido a su actividad antiinflamatoria, antimicrobiana, inmunoestimulante, antioxidante, etc. Se ha realizado diversos estudios sobre la composición química, principalmente en corteza, abundando los estudios en el grupo de los alcaloides. El objetivo de la presente investigación se centra en la cuantificación de polifenoles totales presentes en las hojas, para lo cual se tuvo como material de estudio plantas de un mismo clon establecido en tres localidades de la región de Ucayali, Perú, con condiciones edafoclimáticas diferentes.

Las muestras de hojas se recolectaron en noviembre de 2009, en las localidades de Tres de Octubre, El Porvenir y Nuevo Ucayali, de las provincias de Padre Abad y Coronel Portillo. Previo tratamiento post cosecha, se obtuvo un extracto por el método de percolación utilizando como solventes soluciones hidroalcohólicas en una relación 1:25 de hojas y solvente. Los extractos obtenidos fueron sometidos a pruebas cualitativas (marcha fitoquímica), en las que se detectaron metabolitos como alcaloides, compuestos grasos, flavonoides, azúcares, taninos condensados y saponinas, y también pruebas cuantitativas que permitieron determinar el contenido de polifenoles en el extracto y de taninos condensados presentes en las muestras.

Palabras clave: Uncaria tomentosa, uña de gato, Rubiaceae, polifenoles totales, taninos condensados.

\section{QUANTIFICATION OF POLYPHENOLS IN LEAVES OF CAT'S CLAW Uncaria tomentosa (WILLD. EX SCHULT) D.C. FROM THREE LOCATIONS IN UCAYALI}

\begin{abstract}
Cat's claw (Uncaria tomentosa Willd. ex D.C.), Rubiaceae family, is a vine which is used, traditional and commercially, the crust in several towns in the Peruvian Amazon and other parts of South America: because of their actions antiinflammatory, antimicrobial, immunostimulatory, antioxidant, etc.

This study aimed to quantify total polyphenols present in the leaves of "cat's claw" Uncaria tomentosa from the same clone set in three locations from Ucayali.

The botanical material was collected in November 2009, in Tres de Octubre, El Porvenir and Nuevo Ucayali, covering the districts of Padre Abad Province Irazola and Coronel Portillo, Ucayali department. With the leaf samples was extracted by percolation method using as solvent an alcohol solution in a 1:25 ratio and solvent leaves. The extracts obtained were
\end{abstract}

a Universidad Nacional Agraria La Molina. Facultad de Ciencias Forestales. Lima, Perú. gdominguez@lamolina.edu.pe deysigl@lamolina.edu.pe 
subjected to qualitative tests (phytochemical), which were detected as metabolites: alkaloids, fatty compounds, flavonoids, sugars, condensed tannins and saponins, and allowed to determine the quantitative content of polyphenols in the extract and the percentage condensed tannins present in the samples.

Key words: Uncaria tomentosa, cat's claw, Rubiaceae, polyphenols, condensed tannins.

\section{INTRODUCCIÓN}

El género Uncaria es una fuente importante de productos naturales medicinales, particularmente por el contenido de alcaloides y triterpenos. Durante los últimos 20 años, diversos alcaloides, triterpenos, glicósidos de ácido quinóvico, flavonoides y cumarinas, han sido aislados de este género. La especie identificada con un mayor número de componentes químicos es la "uña de gato" Uncaria tomentosa de Perú

Uncaria tomentosa (Willd.) D.C., ha sido parte de diversas investigaciones en centros especializados de Austria (Universidad de Graz, Innsbruck), Alemania (Instituto de Biología Farmacéutica de la Universidad de Munich), Italia (universidades de Nápoles, Salerno, Pavia, Milan), Perú (Universidad Peruana Cayetano Heredia, Universidad Nacional Mayor de San marcos) y en la Universidad Nacional Agraria La Molina, Universidad de Ucayali, Pontificia Universidad Católica del Perú y Universidad Agraria de la Selva (con énfasis en aspectos agronómicos), entre otras. De acuerdo a investigaciones se ha determinado que esta especie posee propiedades de tipo antiinflamatorio, inmunoestimulante, antioxidante, antimutágena y antiviral.

Aunque los principales compuestos activos, como los alcaloides, han sido estudiados mayormente en la corteza de Uncaria, estos pueden también estar presentes en diferentes partes de la planta ${ }^{3}$. Sin embargo, las propiedades de la uña de gato no se debe exclusivamente a la presencia de alcaloides; existen otros metabolitos secundarios que en conjunto presentan una acción sinérgica, las cuales, como los polifenoles, tienen efectos benéficos sobre el organismo humano. Estos compuestos constituyen un grupo químico importante y se encuentran presentes en muchas plantas medicinales; no necesariamente son esenciales para el metabolismo de la planta pero son sintetizados como mecanismos de defensa contra los predadores ${ }^{4}$, adaptación de la misma y contra la prevención de daños oxidativos ${ }^{5}$. Por ello, se enfatiza en la importancia del estudio de los diferentes compuestos existentes y no en el aislamiento de un solo tipo de metabolito.

Partiendo de esta hipótesis, en el marco del proyecto FINCyT - Pibap 023-2009 "Estandarización de un extracto seco purificado a partir de hojas de Uncaria tomentosa (Willd.) D.C. con fines de formulación farmacológica", desarrollado en la Universidad Nacional Agraria La Molina, se realizó el estudio en hojas de Uncaria tomentosa Willd ex. Schult D.C. proveniente de plantaciones experimentales de un mismo clon producido in vitro y establecidos en tres localidades del departamento de Ucayali, que presentan condiciones de hábitat diferentes. El objetivo del estudio es determinar metabolitos secundarios, cuantificándolos y estableciendo las diferencias en el contenido de poli-fenoles de las hojas con la finalidad de contribuir al conocimiento fitoquímico y su variabilidad en las tres procedencias trabajadas.

\section{Lugar de ejecución}

\section{PARTE EXPERIMENTAL}

El presente trabajo de investigación se realizó en el Laboratorio de Transformación Química de la Madera de la Facultad de Ciencias Forestales de la Universidad Nacional Agraria La Molina. 


\section{Materiales reactivos y equipos}

\section{Muestra}

Las muestras provienen de plantaciones experimentales a campo abierto de un mismo clon de Uncaria tomentosa, ubicadas entre los kilómetros 98 y 203 de la Carretera Federico Basadre (Pucallpa - Lima) en las localidades de: Tres de Octubre, El Porvenir y Nuevo Ucayali, pertenecientes a la cuenca del río Aguaytía, región Ucayali.

\section{Reactivos}

Ácido clorhídrico concentrado, butanol (grado reactivo), etanol 96º acetona p.a, reactivo de Folin Ciocalteu (Merck), solución de carbonato de sodio (20\%), cinta de magnesio, tricloruro férrico, cloruro de sodio sólido p.a, hidróxido de sodio sólido p.a., patrón de ácido tánico (Chromadex), se utilizó agua ultrapura para todos los experimentos.

\section{Equipos}

Hornillas eléctricas (Herhardt), mufla modelo Furnace 48000 (Thermolyne), balanza (SAUTER) de 0,01 g de precisión, balanza analítica (OHAUS) de 0,0001 mg de precisión, estufa de $0^{\circ}-115^{\circ} \mathrm{C}$. modelo KT500 (Heraus), agitador orbital de 0 a 500 revoluciones $/ \mathrm{min}$. (IKA LABORTECHNIK), rotavapor modelo R-210 (BUCHI), espectofotómetro UV - Vis modelo Helios ä (Thermo electron Corporation).

\section{Métodos de análisis}

\section{Ensayos físicos a las hojas de Uncaria tomentosa Humedad gravimétrica}

Se realizó de acuerdo al procedimiento descrito en la NRSP 309, norma cubana ${ }^{6}$. Este ensayo se realiza por el método gravimétrico cuando la droga cruda no contiene sustancias volátiles.

\section{Cenizas totales}

Se realizó el ensayo señalado para: métodos de ensayo en droga cruda para medicamentos de origen vegetal NRSP 309 Ministerio de Salud Pública de Cuba.

\section{Solubilidad}

Consiste en determinar el grado de alcohol del cual los diferentes metabolitos secundarios son más solubles, y el procedimiento está descrito en la norma NRSP - 309. Se hizo una prueba de solubilidad para cada muestra de hojas, con cuatro proporciones diferentes de solvente (agua: etanol) etanol al 30\%, etanol al 50\%, etanol al $70 \%$ y agua. Lo cual indica diferentes grados de polaridad del solvente ${ }^{6}$.

\section{Obtención de los extractos}

Se utilizó el método de re-percolación que consiste en pasar el solvente a través de la muestra vegetal hasta agotamiento de la muestra. El solvente utilizado para las hojas de Uncaria tomentosa fue una solución hidroalcohólica, cuyo porcentaje varía de acuerdo a la solubilidad previamente determinada, para cada una de las muestras; se humedeció la muestra vegetal, para aumentar el contacto con el solvente facilitando el paso del mismo y no permitiendo la formación de falsas vías, que perjudiquen la eficiencia; el humedecimiento se debe realizar fuera del percolador ya que la muestra podría hincharse excesivamente y comprimirse en las paredes del percolador obstaculizando el paso del solvente; seguidamente se dejó reposar por 24 horas, se colectó el primer extracto para luego pasarlo al siguiente percolador, y al primer percolador se le añadió solvente puro, el mismo volumen usado la primera vez y se dejó reposar otras 24 horas. Se realizó el mismo procedimiento para los cinco percoladores colectándose el extracto enriquecido después de seis días.

\section{Caracterización del extracto}

Se evaluó los parámetros de $\mathrm{pH}$, densidad relativa, sólidos totales, polisacáridos totales. 


\begin{abstract}
Análisis fitoquímico
Una vez identificado el grado de alcohol en el que los metabolitos son más solubles mediante el ensayo de solubilidad, se enumeró 12 tubos de ensayo, uno para cada prueba, y en cada uno se colocó aproximadamente $1 \mathrm{~mL}$ del extracto obtenido; luego, se procedió a la identificación de los mismos mediante los diferentes ensayos fitoquímicos, usando los siguientes reactivos: Dragendorff (alcaloides), Mayer (alcaloides), Wagner (alcaloides), Bornträger (quinonas), Sudán (compuestos grasos), Fehling (azúcares reductores), ensayo de la espuma (saponinas), tricloruro férrico - $\mathrm{FeCl}_{3}$ (compuestos fenólicos: taninos), Shinoda (flavonoides), Reactivo de Kedde, gelatina, reactivo de Legal.
\end{abstract}

\title{
Cuantificación de polifenoles totales mediante el método espectrofotométrico ${ }^{7}$ Determinación de la curva estándar
}

Sustancia de referencia: se utilizó ácido tánico p.a, del cual se pesó con precisión 0,5 mg de ácido tánico, se transfirió a una fiola de $500 \mathrm{~mL}$ y se completó el volumen con agua destilada. Blanco: Se añaden $5 \mathrm{~mL}$ de agua destilada a un matraz aforado de $25 \mathrm{~mL}$.

Muestra: Se midió exactamente $2 \mathrm{~mL}$ del extracto y fue transferido a una fiola de $100 \mathrm{~mL}$, diluyendo con agua destilada hasta enrase.

\section{Desarrollo de color}

A cada matraz aforado de $25 \mathrm{~mL}$, con las respectivas muestras, patrones (soluciones de ácido tánico de concentración 0 a 10 ppm, en intervalos de 2ppm) y blanco, se le añadió $2 \mathrm{~mL}$ de solución Folin Ciocalteu, se agitó y se dejó reposar durante 5 min. Luego se añadió $1 \mathrm{~mL}$ de solución de carbonato de sodio al $20 \%$, se agitó, enrasó con agua destilada y homogenizó. Se procedió a leer la absorbancia de dichas soluciones a $760 \mathrm{~nm}$ después de transcurridos 2 minutos y los resultados se expresan en $\mu \mathrm{g}$ equivalentes de ácido tánico/g muestra.

\section{Cuantificación de taninos condensados mediante el método Número de Stiasny ${ }^{8}$}

Se agregó $10 \mathrm{~mL}$ de formaldehído al $37 \%$ y $5 \mathrm{~mL}$ de ácido clorhídrico concentrado a $50 \mathrm{~mL}$ del extracto. Esta mezcla se llevó a ebullición a reflujo durante 30 minutos; el precipitado formado se separó en papel filtro utilizando una bomba de vacío y se lavó con agua caliente para eliminar el ácido; luego se procedió a llevar a sequedad en la estufa y pesar; previamente se determinó la cantidad de sólidos totales en $50 \mathrm{~mL}$ del extracto, evaporándolos a sequedad y pesando.

El Número de Stiasny es la relación entre el precipitado formado respecto a los sólidos totales y corresponde al porcentaje de taninos condensados en el extracto. Este porcentaje se calcula multiplicando este número en fracción por el rendimiento en sólidos obtenidos en cada extracto.

Número de Stiasny $=\left(\mathrm{pp}^{*} 100\right) / \mathrm{pr}$

$$
\mathrm{tc}=(\mathrm{ns} * \mathrm{et}) / 100
$$

Donde:

pp: peso del precipitado $(\mathrm{g})$

pr: peso del residuo de $50 \mathrm{~mL}$ de extracto $(\mathrm{g})$

tc: porcentaje de taninos condensados

ns: $\mathrm{N}^{\circ}$ Stiasny

et: Porcentaje del extracto total 


\section{RESULTADOS Y DISCUSIÓN}

\section{Ensayos físicos}

\section{Humedad gravimétrica}

Los valores de humedad aceptados por la Farmacopea Europea para drogas vegetales tienen como límite superior $12 \%$ de contenido de humedad; por lo tanto los valores hallados en las muestras son los adecuados para trabajar los posteriores análisis (tabla 1).

Tabla 1. Contenido de humedad gravimétrica en hojas de Uncaria tomentosa

\begin{tabular}{ccccc}
\hline Localidad & Muestra & $\begin{array}{c}\text { Humedad } \\
(\%)\end{array}$ & $\begin{array}{c}\text { Humedad } \\
\text { promedio } \\
(\%)\end{array}$ & $\begin{array}{c}\text { Desviación } \\
\text { estándar }\end{array}$ \\
\hline Tres de & S1 & 109,944 & & \\
Octubre & S2 & 109,372 & 112,110 & 0,4256 \\
& S3 & 117,013 & & \\
\hline El & S1 & 101,927 & & \\
Porvenir & S2 & 104,732 & 108,132 & 0,8434 \\
& S3 & 117,735 & & \\
Nuevo & S1 & 96,423 & & \\
Ucayali & S2 & 100,319 & 99,083 & 0,2306 \\
\hline & S3 & 100,508 & & \\
\hline
\end{tabular}

\section{Contenido de cenizas totales}

En las drogas vegetales, según la Real Farmacopea Española, los valores permitidos oscilan entre 5-24\%, pero los valores más frecuentes están entre 8-10\%; según esto, los porcentajes de cenizas encontrados en Uncaria tomentosa, para los tres lugares, se encontrarían dentro de los límites normales para drogas vegetales (tabla 2).

Tabla 2. Contenido de cenizas totales en Uncaria tomentosa

\begin{tabular}{ccccc}
\hline Localidad & Muestra & \% Cenizas & $\begin{array}{c}\text { Promedio } \\
\text { cenizas (\%) }\end{array}$ & $\begin{array}{c}\text { Desv. } \\
\text { estándar }\end{array}$ \\
\hline \multirow{2}{*}{ Tres de } & S1 & 38,025 & & \\
Octubre & S2 & 0,8911 & 30,213 & 18,666 \\
& S3 & 43,704 & & \\
El Porvenir & S1 & 10,774 & & \\
& S2 & 25,257 & 17,866 & 0,7246 \\
& S3 & 17,568 & & \\
Nuevo & S1 & 41,309 & & \\
Ucayali & S2 & 41,933 & 42,295 & 0,1208 \\
\hline & S3 & 43,643 & & \\
\hline
\end{tabular}




\section{Grado de solubilidad}

El grado alcohólico, donde se concentra la mayor cantidad de sólidos, fue variable entre las muestras, de acuerdo a la procedencia (tabla 3). Por tanto, cada extracto realizado posee diferente solubilidad; esto probablemente se deba a la variación en la cantidad de metabolitos presentes en las hojas; un grado de solubilidad alto podría deberse a un mayor contenido de compuestos extraíbles y de características similares al solvente de extracción.

Tabla 3. Resultados del ensayo de solubilidad

\begin{tabular}{cc}
\hline Localidad & \% Solubilidad \\
\hline Nuevo Ucayali & 70 \\
El Porvenir & 50 \\
Tres de Octubre & 30 \\
\hline
\end{tabular}

Análisis fitoquímico: (tabla 4)

Tabla 4. Tamizaje fitoquímico en extractos de hojas de Uncaria tomentosa

\begin{tabular}{|c|c|c|c|}
\hline Ensayo & $\begin{array}{c}\text { Tres de } \\
\text { Octubre }\end{array}$ & El Porvenir & $\begin{array}{c}\text { Nuevo } \\
\text { Ucayali }\end{array}$ \\
\hline Dragendorff & + & + & +++ \\
\hline Mayer & ++ & ++ & ++ \\
\hline Wagner & - & - & - \\
\hline Bornträger & ++ & + & + \\
\hline Sudán & + & + & + \\
\hline Fehling & ++ & + & ++ \\
\hline Espuma & ++ & + & + \\
\hline Cloruro & ++ & ++ & ++ \\
\hline Sérrico & ++ & ++ & ++ \\
\hline Kedde & ++ & + & +++ \\
\hline Gelatina & +++ & ++ & ++ \\
\hline Legal & + & - & - \\
\hline $\begin{array}{l}\text { Leyenda: }+++\mathrm{R} \\
+ \text { Reacción poco }\end{array}$ & $\begin{array}{l}n \text { muy ev } \\
\text { te pero a }\end{array}$ & $\begin{array}{l}++ \text { Rea } \\
\quad-\text { Noh }\end{array}$ & $\begin{array}{l}\text { on evidente } \\
\text { o reacción }\end{array}$ \\
\hline
\end{tabular}

\section{Cuantificación de los polifenoles totales}

Se obtuvo para la localidad de Tres de Octubre 2,24 ppm; para El Porvenir 3,99 ppm, y para Nuevo Ucayali 6,47 ppm, expresado como ácido tánico. De acuerdo a los resultados obtenidos, la localidad de Nuevo Ucayali es la que presenta mayor concentración. Se puede 
significar que las hojas de uña de gato tienen actividad antiinflamatoria ${ }^{1,9,10}$ mayor con respecto a la corteza y podría deberse a la diferente concentración y composición química de las mismas, especialmente a la presencia de los flavonoides que conforman el gran grupo de los polifenoles.

Linealidad del método de cuantificación (tabla 5):

Tabla 5. Test de linealidad para el ácido tánico

\begin{tabular}{|c|c|c|c|c|}
\hline $\mathbf{X}(\mathbf{p p m})$ & $\mathbf{Y}(\mathbf{a b s})$ & $\mathbf{F}(\mathbf{y} / \mathbf{x})$ & \multirow{2}{*}{ Desviación estándar } & \multirow[t]{2}{*}{3,742} \\
\hline 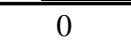 & 0,011 & $0,0,000$ & & \\
\hline 2 & 0,132 & 0,066 & Promedio & 5,000 \\
\hline 4 & 0,234 & 0,059 & Coeficiente de correlación & 0,994 \\
\hline 6 & 0,338 & 0,056 & Ecuación de la recta & $Y=0,034+0,046 \mathrm{X}$ \\
\hline 8 & 0,406 & 0,051 & c.v.f & $0,75 \%$ \\
\hline 10 & 0,476 & 0,048 & Criterio c.v.f & $<5 \%$ \\
\hline
\end{tabular}

c.v.f: coeficiente de variación para el factor de respuesta f

La curva de calibración para la cuantificación de polifenoles totales en los rangos de concentración conocidos, responden a la ecuación: $Y=0,034+0,046 \mathrm{X}$ y tienen un coeficiente de correlación de 0,994. Los factores respuesta en la muestra se mostraron variables de acuerdo a su procedencia. Además, el gráfico "absorbancia versus concentración” de ácido tánico muestra linealidad en la curva de calibración (figura 1).

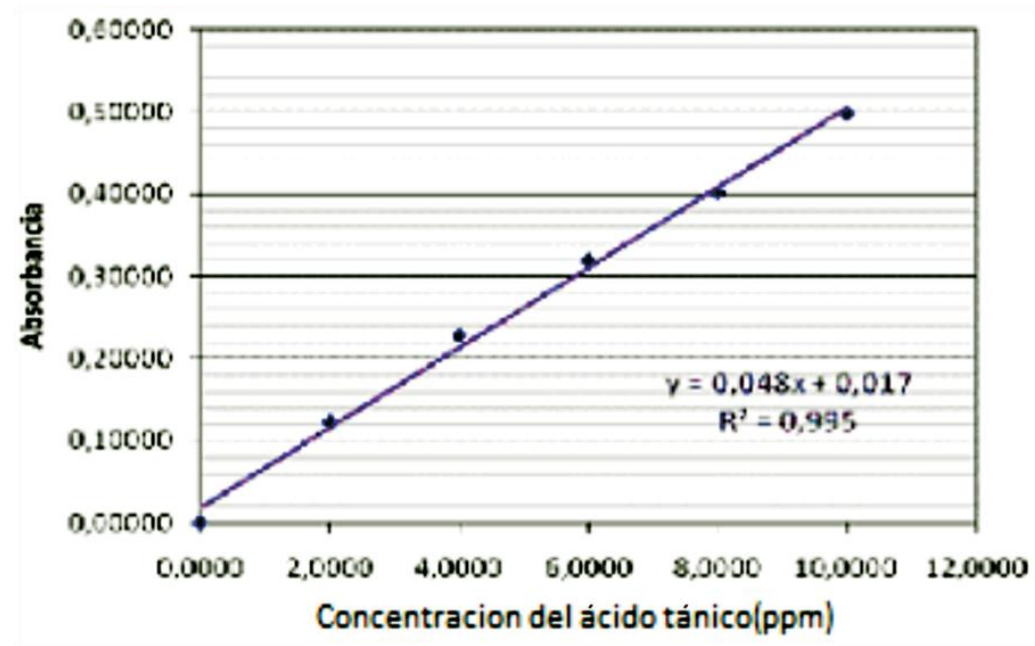

Figura 1. Linealidad para el ácido tánico 


\section{Cuantificación de los taninos condensados}

Se obtuvo para la localidad de Nuevo Ucayali 31,84\%, mientras que para la localidad El Porvenir fue $30,51 \%$ y para la localidad de Tres de Octubre 26,26\% de taninos condensados como observamos en la tabla 6.

Tabla 6. Porcentaje de taninos condensados

\begin{tabular}{lccc}
\hline Localidad & $\begin{array}{c}\text { Rendimiento } \\
\text { del extracto } \\
(\boldsymbol{\%})\end{array}$ & $\begin{array}{c}\mathbf{N}^{\mathbf{0}} \text { de } \\
\text { Stiasny }\end{array}$ & $\begin{array}{c}\text { Taninos } \\
\text { Condensados } \\
\mathbf{( \% )}\end{array}$ \\
\hline El Porvenir & 43,15 & 55 & 25,85 \\
& & 68 & 23,67 \\
Tres de & & 76 & 29,26 \\
Octubre & 38,02 & 82 & 31,31 \\
& & 82 & 31,29 \\
Nuevo Ucayali & 46,34 & 96 & 44,36 \\
& & 52 & 24,26 \\
\hline
\end{tabular}

La presencia de estos taninos condensados en partes aéreas de la planta constituye el mecanismo de defensa y estos podrían actuar como atrapadores de radicales libres y agentes antivirales ${ }^{4}$.

\section{CONCLUSIONES}

El tamizaje fitoquímico realizado permitió la detección de metabolitos en las muestras de hojas de Uncaria tomentosa de las diferentes procedencias. También se comprobó la presencia de: alcaloides, quinonas, flavonoides, taninos pirocatecólicos.

Se tiene como resultado de la cuantificación de polifenoles totales, que las muestras de hojas proveniente de Nuevo Ucayali presentan mayor contenido, expresados como ácido tánico; por otro lado, las muestras provenientes de Tres de Octubre presentan menor contenido de polifenoles totales.

El mayor porcentaje de taninos condensados en hojas de Uncaria tomentosa se obtuvo de las muestras de Nuevo Ucayali.

\section{AGRADECIMIENTOS}

Al Fondo para la Innovación, Ciencia y Tecnología (FINCyT PIBAP 023-2009) 


\section{BIBLIOGRAFÍA}

1. Heitzman, M.; Neto, C.; Winiarz, E; Vaisberg, A. Hammond G. Ethnobotany, phytochemistry and pharmacology of Uncaria (Rubiaceae). Phytochemistry. 2005; 66(5): 5-29.

2. Angulo, P.; Wilder A.; Miguez, M.P.; Cascos, P. Actividad de la hojas y alcaloides de la uña de gato (Uncaria tomentosa Willd DC) en el modelo de inflamación intestinal crónica de Yamada et al. 2007. Artículo en línea, disponible en http://www.samento.co.ec/sciencelib/addins/4ccespn/rv_ArtUnaGato.pdf

3. Domínguez G. Efecto de diferentes hábitats en el rendimiento y la calidad fitoquímica del cultivo clonal de Uncaria tomentosa (Willd.) DC. Tesis (Dr. Sc.) Pinar del Río, CU. Universidad Pinar del Río "Hermanos Saiz Montes de Oca”. 2007.

4. Ricco, R.; Sena, G.; Vai, V.; Wagner, M.; Gurni, A. Taninos condensados de Ephedra chilensis K. presl (=E. Andina Poepp.Ex. May)- Ephedraceae. Revista Dominguezia 2002; 18(1): 17-25.

5. Marcano, D. y Hasegawa, M. Fitoquimica Orgánica. Segunda Edición, Consejo de Desarrollo Científico y Humanístico. Universidad de Venezuela. 2002

6. Norma Ramal de Salud Pública N³09 (NRSP). Medicamentos de origen vegetal, droga cruda. Métodos de ensayo. Ministerio de Salud Pública. La Habana, Cuba. MINSAP. 1992

7. Inocente, M.; Fuentes, C.; Jurado, B. Cuantificación de taninos condensados en Triplaris americana L. (tangarana colorada). Revista de la Sociedad Química del Perú. 2009; 76 (2):138-148.

8. Rosales, M. y Gonzales, R. Comparación del contenido de compuestos fenólicos en la corteza de ocho especies de pino. Madera y Bosques 2003 9(2): 41-49.

9. Giraldo, L.; Hernández, M.; Angulo; Fuertes, C. Actividad antinitrosativa y antiinflamatoria de los flavonoides de las hojas de Uncaria tomentosa Willd. DC. (uña de gato). Revista de la Sociedad Química del Perú; 2003; 69(4); 229-242.

10. León, F. y Cabieses, F. Efecto antiinflamatorio de la Uncaria tomentosa (uña de gato). Odontología Sanmarquina. 2000; 1 (6): 66-68. 